\title{
EFFECT OF DESOXYCORTICOSTERONE GLUCOSIDE UPON CEREBRAL BLOOD FLOW AND METABOLISM OF HUMAN SUBJECTS ${ }^{1}$
}

\author{
By R. C. BENTINCK," GILBERT S. GORDAN, JOHN E. ADAMS, L. H. ARNSTEIN, \\ AND T. B. LEAKE \\ (From the Dizisions of Medicine and Neurological Surgery, Unizersity of California School of \\ Medicine, San Francisco)
}

(Submitted for publication August 8, 1950; accepted, November 13, 1950)

\section{INTRODUCTION}

An effect of steroidal hormones upon cerebral metabolism was suggested by Selye's (1) observation that steroids possess anesthetic properties when administered to experimental animals. Gordan and Elliott (2) subsequently showed that certain steroids inhibit glucose utilization by rat brain in vitro and that their comparative inhibitory activity parallels their reported anesthetic potency. These observations were confirmed by Hayano, Schiller, and Dorfman (3). The possibility that steroidal inhibition of cerebral oxidative processes may be a physiological occurrence was demonstrated by Eisenberg, Gordan, and Elliott $(4,5)$. They found that deprivation of gonadal steroids by castration produces an increase in the rate of glucose utilization by rat brain and that the in vivo administration of testosterone, as well as other steroids with the androstene nucleus, prevents the characteristic rise. Desoxycorticosterone was the most potent of the steroidal compounds tested.

Evidence of an in viz'o action of steroidal hormones on cerebral functions in man may be inferred from the observations of Pincus and Hoagland (6-9). In controlled studies, they found that the administration of pregnenolone to human subjects results in a significant lowering of fatigue responses to psychomotor tests. It is of interest that euphoria, depression, and exhilaration have been reported from the administration of various steroids in man (8-13).

The known anticonvulsive activity of desoxycorticosterone further suggests a steroidal influence on cerebral processes. Woodbury and Daven-

\footnotetext{
1 Aided by a cooperative grant between the National Heart Institute and the University of California and a grant-in-aid from the Research Committee of the University of California.

2 Schering Fellow in Endocrinology, 1949-1950.
}

port (14) have demonstrated that the lowered electro-shock seizure threshold of adrenalectomized animals may be restored to normal levels, and that it may be increased above normal in intact animals by desoxycorticosterone acetate. In human epileptic patients, the anticonvulsive activity of this steroid has also been demonstrated. McQuarrie, Anderson, and Ziegler (15) and Aird and Gordan (16) have reported a reduction in the frequency of convulsive episodes of epileptic patients receiving desoxycorticosterone acetate. Desoxycorticosterone glucoside, an active water-soluble preparation of desoxycorticosterone, has been shown to reduce the electro-encephalographic abnormalities of epileptic patients (16).

These considerations have stimulated further investigation of steroidal activity upon cerebral metabolism in man. There is a suitable method, the nitrous oxide technique of Kety and Schmidt $(17,18)$, for the determination of cerebral blood flow in man. An estimation of the rate of cerebral blood flow permits a computation of the rate of utilization or production by the brain of any substance which can be accurately analyzed in blood. A steroidal compound well adapted for use in short-term studies in man is also available in the potent, water-soluble desoxycorticosterone glucoside (DCG). ${ }^{3}$ The conjunction of a workable method for the estimation of the rate of cerebral blood flow and an active, water-soluble steroid has made feasible a measurement of steroidal effects on the metabolism of the human brain in vivo.

\section{METHOD}

Cerebral blood flow (CBF) and cerebral metabolic rate $(C M R)$ were estimated in 15 human subjects by the original nitrous oxide method of Kety and Schmidt (17,

\footnotetext{
3 The desoxycorticosterone glucoside used in this study was generously furnished by Ciba Pharmaceutical Products, Inc., Summit, New Jersey.
} 
18). Of the patients studied, seven had no endocrine disease, one had mild diabetes mellitus controlled by diet alone, two had been castrated for prostatic carcinoma, one represented Klinefelter's syndrome with hypoleydigism, and four had chromophobe adenomas with secondary hypogonadism. The subjects received no medications for 24 hours or more before the study and hormonal medications had been withheld for the preceding two or more weeks.

With the fasting subject supine, needles were inserted into the jugular bulb and either the femoral or internal carotid artery and connected to heparinized manifolds as described by Kety and Schmidt. In order to avoid the effects of emotional stimuli, as stressed by Scheinberg and Stead (19), no further procedures were undertaken until a steady state was achieved as determined by stable cardiac rate and blood pressure. In 14 of the 15 patients, two determinations of the rate of cerebral blood flow were made consecutively. Twenty minutes were allowed to elapse after the control determination to permit expiration of nitrous oxide, and a second blood blank for nitrous oxide was obtained in each case. Prior to proceeding with the second cerebral blood flow determination, specimens for control glucose levels of arterial, cerebral venous and, in seven cases, peripheral (antecubital) venous blood were obtained. All of these were drawn simultaneously at two-minute intervals until three separate sets of control specimens had been obtained.

Immediately after the third set of control samples for glucose determination were taken, $50 \mathrm{mgm}$. of DCG were injected intravenously and a second determination of the cerebral blood flow was made without delay. Blood sugar values were determined on aliquots of the simultaneously drawn experimental specimens of arterial and cerebral venous blood throughout the procedure for comparison with the previously drawn control specimens. Following completion of the second cerebral blood flow determination, two additional sets of specimens were obtained for analysis of sugar levels at three-minute intervals. When peripheral (antecubital) venous specimens were obtained, they were drawn simultaneously with the arterial and cerebral venous specimens. During each cerebral blood flow determination, arterial and cerebral venous oxygen and carbon dioxide content was determined upon separate specimens consisting of $5 \mathrm{cc}$. of blood drawn immediately before the first CBF sample and an equal amount drawn into the same syringe directly following the five-minute $\mathrm{CBF}$ specimen.

Blood oxygen and carbon dioxide analyses were made in duplicate by the manometric technique of Van Slyke and Neill (20), as modified by Kety and Schmidt (18). Blood sugar content was determined by the iodometric titration method of Somogyi $(21,22)$ and yielded results reproducible within 1 per cent. In accordance with the injunction of Somogyi (23) that, when dealing with changes in arterio-venous glucose difference, accurate analytical methods must be selected and employed with meticulous care, all glucose determinations were performed in duplicate by one of us (R.C.B.). Mean arterial blood pressure was obtained directly from the femoral or carotid artery by means of an air-damped mercury manometer connected to the arterial manifold. Cerebral oxygen consumption, cerebral glucose consumption or production, and cerebral carbon dioxide production were calculated from the rate of cerebral blood flow by the formulae of Kety and Schmidt.

\section{EXPERIMENTAL DATA}

Experimental data are presented in Table $I$. For each function measured, the mean value and the standard deviation of the mean is included.

Striking and rapid effects were noted upon blood sugar levels following administration of 50 mgm. of DCG. Uniformly, the cerebral venous glucose concentration rose significantly $\rightarrow$ eight instances above the arterial level-effecting in 13 of the 15 cases a reduction in the cerebral arteriovenous glucose difference. While the individual changes in the arterial and peripheral (antecubital) venous blood sugar concentrations were inconsistent and not marked, a small, but statistically significant $(p<0.01)$, rise occurred in both the mean arterial and the mean antecubital venous blood sugar concentrations following the administration of DCG. Examples of the glucose concentration curves are shown in Figures 1 and 2. It should be noted that the changes in glucose concentration occurred without alteration in the rate of cerebral blood flow. Figure 3 depicts the change in mean cerebral arterio-venous glucose difference produced by DCG. This demonstrates

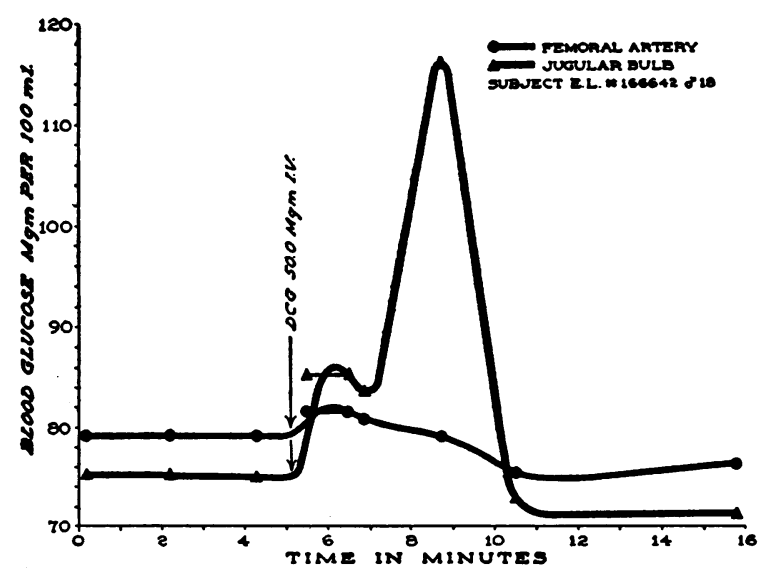

Fig. 1. Effect of DCG on Cerebral Venous and Arterial Glucose Concentrations

Note that the cerebral venous sugar level rises rapidly above the arterial glucose level and remains elevated for approximately five minutes. 


\begin{tabular}{|c|c|c|c|c|c|c|c|c|c|c|c|c|c|c|c|c|c|}
\hline z & 畐 & 2 & & & $\mp:$ & $a$ & & & & $\approx$ & & & 10 & & 10 & |cin & \\
\hline 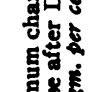 & 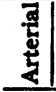 & $\begin{array}{l}N \\
\end{array}$ & $\approx$ & $\stackrel{+}{\rightarrow}$ & 0. & $\infty$ & $\stackrel{0}{\rightarrow}$ & $\stackrel{*}{*}$ & $\begin{array}{l}m \\
-\end{array}$ & 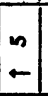 & $\vec{\Rightarrow}$ & $a$ & $\infty$ & $\dddot{2}$ & $\approx$ & 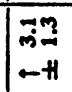 & \\
\hline 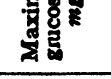 & 3 & 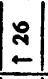 & $\exists$ & $\infty$ & 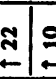 & $\infty$ & $m$ & $:$ & $=$ & $n$ & $\stackrel{7}{\square}$ & $\underset{\pi}{\pi}$ & 10 & $\dddot{2}$ & 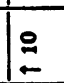 & 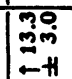 & \\
\hline 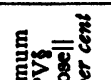 & $\begin{array}{l} \\
\vdots \\
\end{array}$ & \pm & & & $\frac{1}{4}$ & $\mid \begin{array}{l}0 \\
+ \\
+\end{array}$ & & & & \pm & & & 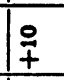 & & $\begin{array}{l}* \\
+\end{array}$ & 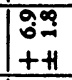 & \\
\hline एव & $\begin{array}{l}0 \\
0 \\
0\end{array}$ & $\begin{array}{l}n \\
+ \\
\end{array}$ & & & $\frac{1}{\mp}$ & $\begin{array}{l}0 \\
+\end{array}$ & & & & \pm & & & in & & \pm & $\mid \begin{array}{l}\text { mo } \\
\vdots 0 \times+1 \\
+H\end{array}$ & \\
\hline 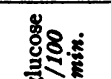 & \begin{tabular}{l|l|l|l|} 
\\
\end{tabular} & $\mid \begin{array}{l}0 \\
1\end{array}$ & $i$ & 11 & $\begin{array}{l}m \\
1 \\
1\end{array}$ & 1 & $\begin{array}{l}n \\
t \\
+\end{array}$ & $i$ & & & $\stackrel{2}{1}$ & $\begin{array}{l}\infty \\
1\end{array}$ & I & i & $\begin{array}{l}n \\
+ \\
+\end{array}$ & 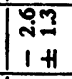 & \\
\hline हैy & $\begin{array}{l}\overline{8} \\
\overline{0} \\
\end{array}$ & $\begin{array}{l}m \\
+\end{array}$ & $\mp$ & \pm & \begin{tabular}{l|l}
0 \\
+ \\
+
\end{tabular} & + & $\frac{\%}{7}$ & in & 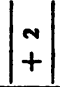 & & $\tilde{t}$ & $1+$ & $\bar{t}$ & + & $i$ & $\begin{array}{l}F \infty \\
+\infty \\
++1\end{array}$ & \\
\hline 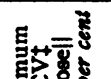 & $\begin{array}{l}0 \\
\\
\end{array}$ & $\mid \frac{a}{i}$ & $\begin{array}{l}n \\
1\end{array}$ & $\begin{array}{ll}m & \\
1 & \\
\end{array}$ & \begin{tabular}{l|l}
0 & $m$ \\
1 & 1 \\
\end{tabular} & \pm & $\overline{7}$ & $i$ & $\left|\begin{array}{l}1 \\
+\end{array}\right|$ & $\mid$ & 市 & a & n & $i$ & $\begin{array}{l}a \\
+ \\
\end{array}$ & 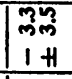 & \\
\hline 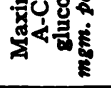 & $\begin{array}{l}\overrightarrow{\mathrm{g}} \\
\mathrm{o} \\
\mathrm{o}\end{array}$ & +1 & \pm & $\begin{array}{l}a \\
+\end{array}$ & $\frac{m}{7}+$ & $\vdots$ & $\nsubseteq$ & $\begin{array}{l}0 \\
+\end{array}$ & $\left|\begin{array}{l}m \\
+ \\
+\end{array}\right|$ & + & $\dot{t}$ & 10 & 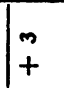 & m & $\dddot{q}$ & $\mid$ & \\
\hline $8 \overline{0}$ & 㗭 & $\mid \stackrel{0}{-1}$ & $:$ & $\stackrel{i}{i}$ & $\overline{\mathrm{i}} \mid \mathrm{m}$ & $\infty_{\infty}^{\infty}$ & $\mid \tilde{\text { I }}$ & 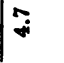 & & & 7 & | & 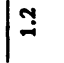 & 13 & iे & 我萧 & \\
\hline 청 & 悹 & $m$ & $=$ & :ִ: & i. & 9 & $\mid \begin{array}{l}m \\
m\end{array}$ & $\hat{m}$ & 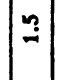 & & ले & $\vec{m}$ & $\Xi$ & 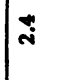 & $\overline{\mathrm{i}}$ & 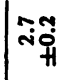 & \\
\hline 8 & \begin{tabular}{l|l} 
\\
0
\end{tabular} & i & $\dot{m}$ & $: 5$ & $\begin{array}{c}\infty \\
\vdots \\
i\end{array} \mid=$ & $\vec{m}$ & 10 & is. & $\mid$ & i⿱日十) & $y$ & $m$ & $\stackrel{\infty}{i}$ & $\stackrel{P}{i}$ & No & $\vec{b} \dot{0}$ & \\
\hline$\frac{8}{80}$ & $\begin{array}{l}\overrightarrow{0} \\
\overline{0} \\
0\end{array}$ & $\mid$ & $\dot{m}$ & $:$ & $\because \mid \begin{array}{l}\infty \\
\infty\end{array}$ & $m_{m}^{\infty}$ & $a$ & $\overrightarrow{0}$ & ì & $\dot{i}$ & i̊ & $m$ & $\ddot{m}$ & $\stackrel{\infty}{m}$ & 官 & 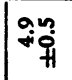 & \\
\hline 㕁 & $\begin{array}{l}0 \\
0 \\
\end{array}$ & 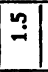 & 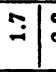 & ip: & $i^{2}$ & $\infty_{\infty}^{\infty}$ & $\overline{\mathbf{N}}$ & 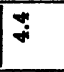 & & & $\stackrel{m}{i}$ & $\vec{m}$ & $\stackrel{i}{i}$ & F & $\stackrel{\infty}{\sim}$ & 冓并 & 8 \\
\hline 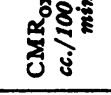 & & $m$ & 9 & 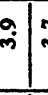 & 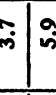 & 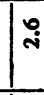 & i. & $\mid \begin{array}{c}\infty \\
\dot{m}\end{array}$ & $\exists$ & & $\hat{\mathrm{j}}$ & $m$ & $\stackrel{n}{n}$ & $\stackrel{i}{i}$ & $\stackrel{n}{\pi}$ & mion & \\
\hline 哭 & $\begin{array}{l}0 \\
\vdots \\
\end{array}$ & $\$$ & $\ddot{0}$ & $=$ & r. & ion & 13 & $\mid \stackrel{0}{i}$ & $\left|\begin{array}{l}0 \\
\dot{m}\end{array}\right|$ & iे & 品 & $\hat{m}$ & $\stackrel{\infty}{*}$ & $\vec{i}$ & 9 & 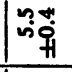 & 鄫 \\
\hline 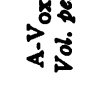 & $\begin{array}{l}\vec{z} \\
\bar{d} \\
0\end{array}$ & $\%$ & is & : & : & F & |ä: & 10 & i & :1 & $\overline{5}$ & 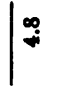 & $\infty$ & 7 & 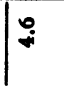 & 战品 & \\
\hline בू: & \begin{tabular}{l|l|l|} 
\\
$\vdots$
\end{tabular} & 2 & & 815 & 815 & 18 & $\mid \ddot{\varpi}$ & $\tilde{E}$ & 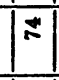 & & 8 & & $1 \%$ & 10 & 18 & $F$ & \\
\hline 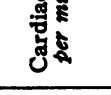 & & 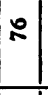 & $\approx$ & \pm & $8: 5$ & $\infty$ & \#ే & R & 8 & & 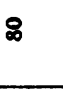 & 18 & 8 & $\approx$ & 2 & $x_{n}$ & \\
\hline 畐产 & $\begin{array}{l} \\
\\
0\end{array}$ & 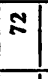 & 815 & 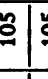 & $=\infty$ & ฮั & 18 & 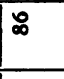 & 18 & & 8 & & 18 & 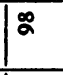 & $\underline{\underline{m}}$ & $\begin{array}{r}\vec{a} a \\
+1\end{array}$ & \\
\hline$\$$ & & $\stackrel{\infty}{\circ}$ & 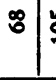 & 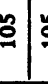 & $=\infty$ & 番 & 8 & $\infty$ & 8 & & ฉ & 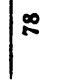 & $\because$ & $\approx$ & i̊n & $\begin{aligned} 8 a \\
H\end{aligned}$ & \\
\hline . & $\begin{array}{l}0 \\
\vdots \\
\end{array}$ & 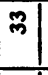 & $\mathrm{N}$ & 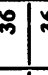 & 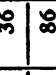 & 10 & |ల్m & $\infty$ & & & 8 & 10 & 7 & $\overline{0}$ & in & so & \\
\hline ชิ & $\begin{array}{l}\bar{z} \\
\bar{z} \\
\end{array}$ & 8 & क) & $5:$ & $=8$ & is & $\infty$ & 8 & in & & ns & $=$ & $\$$ & $\approx$ & $m$ & $\mid \begin{array}{c}\infty_{n} \\
H\end{array}$ & 8 \\
\hline 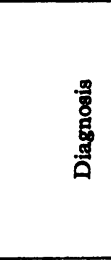 & & 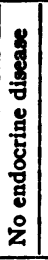 & 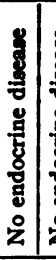 & 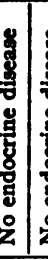 & 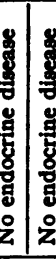 & $\angle$ & 苟 & 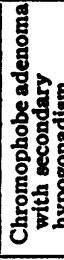 & 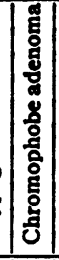 & & 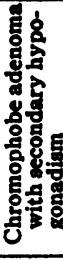 & 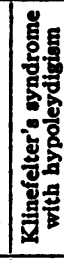 & 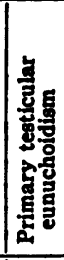 & 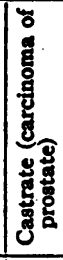 & 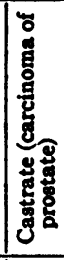 & 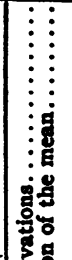 & 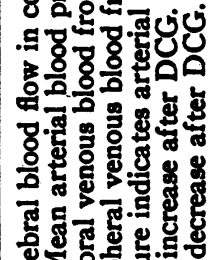 \\
\hline 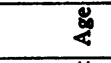 & & 5 & $=1:$ & 919 & 912 & 17 & in & in & $|\bar{n}|$ & 51 & $\Phi$ & $\pi$ & 9 & 18 & 5 & 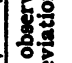 & Un \\
\hline 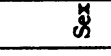 & & 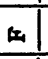 & \pm 10 & $1=$ & $\pm 1 \pm$ & $1 z$ & 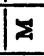 & \pm & $\mid$ & \pm & $\Sigma$ & $\bar{z}$ & $\bar{x}$ & 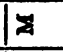 & \pm & 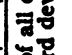 & 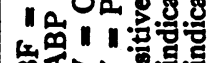 \\
\hline 实葛 & & $\begin{array}{l}\dot{0} \\
\dot{*}\end{array}$ & 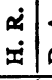 & il & \begin{tabular}{l|l}
3 \\
$\vdots$
\end{tabular} & $\dot{z}_{\dot{x}}^{\dot{z}}$ & \begin{tabular}{|l|}
$x$ \\
$\dot{x}$ \\
\end{tabular} & i & $\left|\begin{array}{l}\dot{\alpha} \\
\dot{x}\end{array}\right|$ & ذا & ند & $\begin{array}{l}\dot{3} \\
\dot{\alpha}\end{array}$ & 迹 & 蛊 & $\begin{array}{l}4 \\
4 \\
\end{array}$ & 照 & \\
\hline$\dot{0}$ & & -1 & 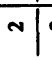 & 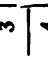 & $-1 n$ & ๑ & - & $\infty$ & $|a|$ & 9 & $=$ & $\simeq$ & $m$ & \pm & $\simeq$ & & \\
\hline
\end{tabular}




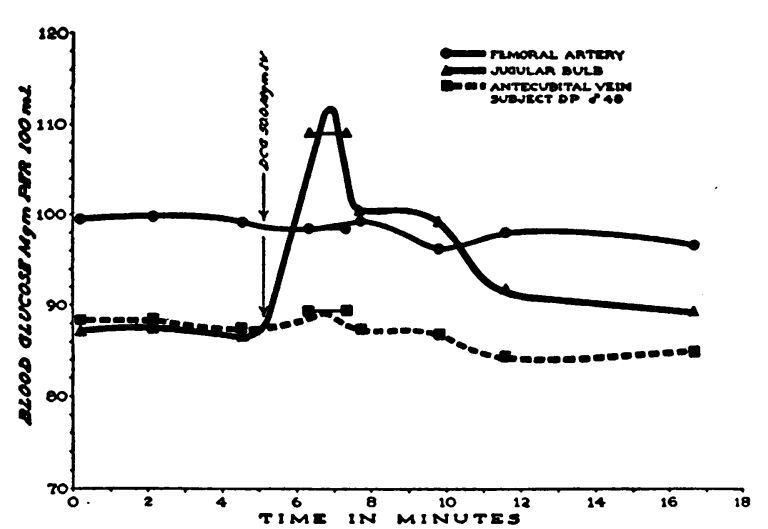

Fig. 2. Effect of DCG on Sugar Content of Arterial and on Cerebral and Peripheral Venous Blood

Note prompt rise of cerebral venous sugar level above arterial glucose concentration in contrast to stable peripheral venous glucose level.

an increase of the mean cerebral venous glucose concentration above the mean arterial glucose concentration after administration of DCG, and, in conjunction with the statistically significant change in $\mathrm{CMR}_{\text {glucose, }}$ is interpreted to indicate liberation of glucose from the brain."

The mean rates of cerebral blood flow, oxygen consumption, and carbon dioxide production did not change significantly. As previously noted by Green and his associates (24), DCG did not alter the mean arterial blood pressure or cardiac rate. No immediate or subsequent clinical effects were apparent.

\section{DISCUSSION}

These experimental findings lend the support of quantitative measurements in man to the thesis that steroidal hormones may affect cerebral carbohydrate metabolism. The consistently observed increase in cerebral venous glucose concentration, with a decrease in the cerebral arterio-venous glucose difference produced by DCG in the absence of increased cerebral blood flow, could have resulted from either diminished cerebral utilization of glucose, liberation of sugar by the brain, or both. An increase in the sugar concentration of the

4 As performed, the Somogyi method is a measure of reducing substance content, almost exclusively monosaccharides. Subsequent to the preparation of this report, we have found that the bulk of the reducing substance liberated into the cerebral venous blood is non-fermentable, and preliminary attempts at identification suggest galactose. cerebral venous blood above that of arterial blood obtained simultaneously indicates liberation of sugar by the brain. From these observations alone, however, it is not possible to conclude that there is not also an alteration of the cerebral utilization of glucose.

From the present data, the mechanism and specificity of this effect may be only conjectured. Of the several possible sources for the sugar liberated from the brain following DCG, a derivation from intracellular glycogen stores may be considered. Kerr and Ghantus (25) have demonstrated sufficient quantities of glycogen in the brains of dogs and rabbits to account for the amount of sugar "liberated" following injection of DCG. The presence of some endogenous substrate in human brain which can be utilized for cerebral oxidative processes in vitro is suggested by Elliott, Sutherland, and Boldrey (26). They found that the oxygen consumption of slices of human cerebral cortex was maintained for a significantly longer period in the absence of a glucose substrate than was the oxygen consumption of rat brain, which dropped off rapidly. If this substrate is glycogen, the glycogenolytic potentiality of desoxycorticosterone reported by Verzar and Wenner (27) would be in harmony with our observations.

Other sources must also be considered, among them the intracellular and extracellular glucose

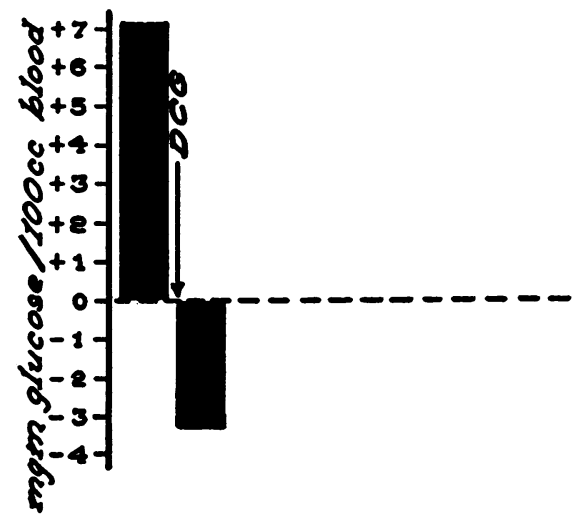

Fig. 3. Cerebral Arterio-Venous Glucose Dimperence: Mean Values for 15 Subjects before DCG and at the Peak Response after DCG

Positive value before DCG indicates that the arterial glucose concentration is higher than the cerebral venous glucose level (glucose utilization). Negative value after DCG indicates that the cerebral venous glucose level excoeds the arterial concentration (glucose "liberation"). 
and cerebral galactosides, but data regarding these are not yet available. Further studies to extend these observations and their specificity are in progress.

No significant alteration in the mean $\mathrm{CMR}_{\mathrm{O}}$, or $\mathrm{CMR}_{\mathrm{CO}}$, was observed, indicating that desoxycorticosterone glucoside does not influence the aerobic phase of cerebral energy metabolism as an immediate general effect, although it may alter the substrate. However, it is interesting that the cerebral metabolic rate was diminished following administration of DCG in all but one ortho-steroidal subject and that no change or only a slight increase in cerebral metabolic rate was apparent in all hyposteroidal subjects. Until the individual groups are expanded, it is not possible to establish the significance of this observation. In this connection it is recognized that the data included in the present paper group together observations made upon individuals with divergent endocrine states. The data suggest that enlargement of the groups may eliminate some of the variability due to the lumping together of non-comparable individuals. The fact that the standard deviations of the mean in this study are somewhat greater than those reported by other workers may, in part, be explained on this basis.

While the arterio-venous sugar difference reflected in the cerebral circulation is altered, no such change occurs in the peripheral arteriovenous glucose difference, suggesting the absence of an immediate effect of desoxycorticosterone glucoside upon the glucose metabolism of the muscular tissues of the forearm. The small but significant rise in the mean arterial glucose concentration following administration of DCG might be accounted for by liberation of glucose from other sources, possibly the liver.

\section{SUM MARY}

1. The effects of desoxycorticosterone glucoside upon the cerebral utilization of oxygen and glucose and the production of carbon dioxide in human subjects has been studied by the nitrous oxide technique of Kety and Schmidt.

2. Administration of DCG produced a rise in the cerebral venous sugar concentration above the arterial level, indicating that this compound causes liberation of sugar from the brain.
3. A small, but significant, increase in arterial glucose concentration also occurred, suggesting liberation of glucose from other sources, possibly the liver.

4. Preliminary data suggest a different response to DCG between individuals with normal and deficient steroidal status.

5. The mean arterial blood pressure, cardiac rate, and rate of cerebral blood flow were not altered by DCG.

\section{REFERENCES}

1. Selye, H., Anesthetic effect of steroid hormones. Proc. Soc. Exper. Biol. \& Med., 1941, 46, 116.

2. Gordan, G. S., and Elliott, H. W., The action of diethylstilbestrol and some steroids on the respiration of rat brain homogenates. Endocrinology, 1947, 41, 517.

3. Hayano, M., Schiller, S., and Dorfman, R. I., Influence of various steroids on the oxidative function of rat tissue preparations. Endocrinology, 1950, 46, 387.

4. Eisenberg, E., Gordan, G. S., and Elliott, H. W., The effect of castration and of testosterone upon the respiration of rat brain. Science, 1949, 109, 337.

5. Eisenberg, E., Gordan, G. S., and Elliott, H. W., Effect of various steroids on aerobic respiration of the brain of the castrate male rat. Federation Proc., 1950, 9, 269.

6. Hoagland, $H$., Adventures in biological engineering. Science, 1944, 100, 63.

7. Pincus, G., and Hoagland, H., Effects of administered pregnenolone on fatiguing psychomotor performance. J. Aviation Med., 1944, 15, 98.

8. Pincus, G., and Hoagland, H., Effects on industrial production of the administration of $\Delta 5$ pregnenolone to factory workers. I. Psychosom. Med., 1945, $7,342$.

9. Pincus, G., Hoagland, H., Wilson, C. H., and Fay, N. J., Effects on industrial production of the administration of $\Delta 5$ pregnenolone to factory workers. II. Psychosom. Med., 1945, 7, 347.

10. Hench, P. S., Kendall, E. C., Slocumb, C. H., and Polley, H. F., The effect of a hormone of the adrenal cortex (17-hydroxy-11-dehydrocorticosterone: Compound E) and of pituitary adrenocorticotrophic hormone on rheumatoid arthritis. Proc. Staff Meet., Mayo Clinic, 1949, 24, 181.

11. Sprague, R. G., Power, M. H., Mason, H. L., Albert, A., Mathieson, D. R., Hench, P. S., Kendall, E. C., Slocumb, C. H., and Polley, H. F., Observations on the physiologic effects of cortisone and ACTH in man. Arch. Int. Med., 1950, 85, 199.

12. Hoagland, H., and Pincus, G., Pituitary-adrenocortical function in patients ${ }^{\circ}$ with severe personality disorders. Proc. First Clin. ACTH Conf., 1950, 1, 544. 
13. Lewin, E., and Wassén, E., Effect of combined injections of deoxycortone acetate and ascorbic acid on rheumatoid arthritis. Lancet, 1949, 2, 993.

14. Woodbury, D. M., and Davenport, V. D., Brain and plasma cations and experimental seizures in normal and desoxycorticosterone-treated rats. Am. J. Physiol., 1949, 157, 234.

15. McQuarrie, I., Anderson, J. A., and Ziegler, M. R., Observations on the antagonistic effects of posterior pituitary and corticoadrenal hormones in the epileptic subject. J. Clin. Endocrinol., 1942, 2, 406.

16. Aird, R. B., and Gordan, G. S., The anticonvulsive properties of desoxycorticosterone. J.A.M.A. (in press).

17. Kety, S. S., and Schmidt, C. F., The determination of cerebral blood flow in man by the use of nitrous oxide in low concentrations. Am. J. Physiol., 1945, 143, 53.

18. Kety, S. S., and Schmidt, C. F., The nitrous oxide method for the quantitative determination of cerebral blood flow in man: theory, procedure and normal values. J. Clin. Invest., 1948, 27, 476.

19. Scheinberg, P., and Stead, E. A., Jr., The cerebral blood flow in male subjects as measured by the nitrous oxide technique. Normal values for blood flow, oxygen utilization, glucose utilization, and peripheral resistance, with observations on the ef- fect of tilting and anxiety. J. Clin. Invest., 1949, 28, 1163.

20. Peters, J. P., and Van Slyke, D. D., Quantitative Clinical Chemistry. Williams \& Wilkins, Baltimore, 1946, Vol. I, 2nd edition.

21. Somogyi, M., A new reagent for the determination of sugars. J. Biol. Chem., 1945, 160, 61.

22. Somogyi, M., Determination of blood sugar. J. Biol. Chem., 1945, 160, 69.

23. Somogyi, M., Studies of arteriovenous differences in blood sugar. II. Effect of hypoglycemia on the rate of extrahepatic glucose assimilation. J. Biol. Chem., 1948, 174, 597.

24. Green, D. M., Johnson, A. D., Bridges, W. C., Lehmann, J. H., and Gray, F., Effects of desoxycorticosterone glucoside on cardiac and renal function. Federation Proc., 1950, 9, 278.

25. Kerr, S. E., and Ghantus, M., The carbohydrate metabolism of brain. II. The effect of varying the carbohydrate and insulin supply on the glycogen, free sugar, and lactic acid in mammalian brain. J. Biol. Chem., 1936, 116, 9.

26. Elliott, H. W., Sutherland, V. C., and Boldrey, E. B., Respiration of human brain slices and the effects of morphine and methadon on oxygen uptake. Federation Proc., 1949, 8, 288.

27. Verzar, F., and Wenner, V., The influence in vitro of desoxycorticosterone on glycogen formation in muscle. Biochem. J., 1948, 42, 35. 